Supporting Information

\title{
Numerical Simulations of Gas-Liquid Two-Phase Flow in Vertical Pipe Implementing Population Balance Modeling
}

\author{
Dhiraj A. Lote ${ }^{1}$, Vadakanchery Vinod ${ }^{2}$, Ashwin W. Patwardhan ${ }^{1 *}$ \\ ${ }^{1}$ Department of Chemical Engineering, Institute of Chemical Technology, Matunga, \\ Mumbai-400019, India \\ ${ }^{2}$ Indira Gandhi Center for Atomic Research, Kalpakkam, Tamil nadu-603102, India \\ dhiraj.lote@gmail.com,aw.patwardhan@ictmumbai.edu.in
}

*Corresponding author: Tel: 91-22-33612018; Fax: 91-22-33611020

Email address: aw.patwardhan@ictmumbai.edu.in 
Table S1: Advantages and limitations of different methods of solving the population balance equation

\begin{tabular}{|l|l|l|}
\hline Methods & Advantage & Disadvantage \\
\hline Class Method & $\begin{array}{l}\text { High accuracy } \\
\text { Predicts particle size distribution directly }\end{array}$ & $\begin{array}{l}\text { High computational cost } \\
\text { Requires a large number of classes }\end{array}$ \\
\hline MUSIG & $\begin{array}{l}\text { High accuracy } \\
\text { Predicts particle size distribution directly }\end{array}$ & $\begin{array}{l}\text { High computational cost } \\
\text { Requires a large number of size groups }\end{array}$ \\
\hline MOM & $\begin{array}{l}\text { Reduces the dimensionality of the problem significantly } \\
\text { Relatively simple to solve }\end{array}$ & Does not provide exact particle size distribution \\
\hline QMOM & Wide range of bubble sizes with reduced computational cost & Does not provide exact particle size distribution \\
\hline
\end{tabular}


Table S2: Summery of previous literature on simulation of gas-liquid two-phase flow for population balance model

\begin{tabular}{|c|c|c|c|c|c|c|c|c|c|c|}
\hline $\begin{array}{l}\text { Author/ } \\
\text { year }\end{array}$ & $\begin{array}{l}\text { Geometry } \\
(\mathrm{mm})\end{array}$ & $\begin{array}{l}\text { Flow } \\
\text { Patterns }\end{array}$ & $\begin{array}{l}\text { Superficial } \\
\text { Velocities }(\mathrm{m} / \mathbf{s})\end{array}$ & CFD Code & Approach & $\begin{array}{l}\text { Turbulence } \\
\text { model }\end{array}$ & Method & $\begin{array}{c}\text { Interfacial } \\
\text { forces/Models }\end{array}$ & $\begin{array}{l}\text { Coalescence } \\
\text { model }\end{array}$ & $\begin{array}{l}\text { Breakage } \\
\text { model }\end{array}$ \\
\hline $\begin{array}{l}\text { Dave et al., } \\
(2017)^{1}\end{array}$ & $\begin{array}{l}\text { Pipe } \\
D=52.3 \\
L=8000\end{array}$ & $\begin{array}{l}\text { Bubbly } \\
\text { and Slug }\end{array}$ & $\begin{array}{l}J_{g}=0.01-0.2 \\
J_{l}=0.5\end{array}$ & & & & IATE & & Fu-Ishii & Fu-Ishii \\
\hline $\begin{array}{l}\text { Yuan et al., } \\
(2016)^{2}\end{array}$ & $\begin{array}{l}\text { Pipe } \\
D=15 \\
L=2500\end{array}$ & Bubbly & $\begin{array}{l}J_{g}=0.1886 \\
J_{l}=2.8294\end{array}$ & CFX-14.5 & Eulerian & $\mathrm{k}-\varepsilon$ & MUSIG & $\begin{array}{l}C_{D} \text { - Ishii-Zuber } \\
C_{L} \text { - Tomiyama } \\
C_{W L}-\text { Antal } \\
C_{T D}-0.1\end{array}$ & $\begin{array}{l}\text { Prince and } \\
\text { Blanch }\end{array}$ & $\begin{array}{l}\text { Neglected due } \\
\text { to small pipe } \\
\text { diameter }\end{array}$ \\
\hline $\begin{array}{l}\text { Pena- } \\
\text { Monferrer } \\
\text { et al., } \\
(2016)^{3}\end{array}$ & $\begin{array}{l}\text { Pipe } \\
D=52 \\
L=5500 \\
D=50.8 \\
L=3000 \\
D=25 \\
L=2000\end{array}$ & Bubbly & $\begin{array}{l}J_{g}=0.02-0.30 \\
J_{l}=1-8\end{array}$ & OpenFOAM & Eulerian & $\mathrm{k}-\varepsilon$ & QMOM & $\begin{array}{l}C_{D}-\text { Schiller-Naumann, } \\
\quad \text { Tomiyama, } \\
\text { Dijkhuizen, } \\
\text { Mei } \\
C_{L}-\text { Wang } \\
C_{W L}-\text { Antal } \\
C_{T D}-\text { Lopez de Bertodano } \\
C_{V M}-0.5\end{array}$ & $\begin{array}{l}\text { Coulaloglou } \\
\text { and } \\
\text { Tavlarides, } \\
\text { Petitti }\end{array}$ & $\begin{array}{l}\text { Narsinhan, } \\
\text { Alopaeus, } \\
\text { Laakkonen and } \\
\text { Alopaeus, } \\
\text { Andersson and } \\
\text { Andersson }\end{array}$ \\
\hline $\begin{array}{l}\text { Swiderski } \\
\text { et al., } \\
(2016)^{4}\end{array}$ & $\begin{array}{l}\text { Pipe } \\
D=50.8 \\
L=3060 \\
D=67 \\
L=6000 \\
\text { Bubble } \\
\text { column } \\
D=200 \\
L=1800\end{array}$ & Bubbly & $\begin{array}{l}J_{g}=0.0275-0.1130 \\
J_{l}=0.25-0.986\end{array}$ & TransAT & & $\mathrm{k}-\varepsilon$ & & $\begin{array}{l}C_{D}-\text { Tomiyama } \\
C_{L}-\text { Tomiyama } \\
C_{W L}-\text { Antal }\end{array}$ & $\begin{array}{l}\text { Coulaloglou } \\
\text { and Tavlarides }\end{array}$ & Laakkonen \\
\hline $\begin{array}{l}\text { Deju et al., } \\
(2015)^{5}\end{array}$ & $\begin{array}{l}\text { Pipe } \\
D=195.3 \\
L=9000\end{array}$ & Bubbly & $\begin{array}{l}J_{g}=0.140-0.2194 \\
J_{l}=1.017\end{array}$ & $\begin{array}{l}\text { Ansys-CFX- } \\
12\end{array}$ & Eulerian & & MUSIG & $C_{L}-$ Tomiyama & $\begin{array}{l}\text { Coulaloglou } \\
\text { and } \\
\text { Tavlarides, } \\
\text { Prince and } \\
\text { Blanch, } \\
\text { Lehr }\end{array}$ & $\begin{array}{l}\text { Luo and } \\
\text { Svendson, } \\
\text { Wang, } \\
\text { Martinez Bazan }\end{array}$ \\
\hline $\begin{array}{l}\text { Schlegel et } \\
\text { al., }(2015)^{6}\end{array}$ & $\begin{array}{l}\text { Pipe } \\
D=304 \\
D=152\end{array}$ & $\begin{array}{l}\text { Bubbly } \\
\text { and cap } \\
\text { bubbly }\end{array}$ & $\begin{array}{l}J_{g}=\text { up to } 11 \\
J_{l}=\text { up to } 2\end{array}$ & & & & IATE & $C_{D}$ - Ishii-Zuber & Smith & Smith \\
\hline
\end{tabular}




\begin{tabular}{|c|c|c|c|c|c|c|c|c|c|c|}
\hline $\begin{array}{l}\text { Wang and } \\
\text { Sun }(2014)^{7}\end{array}$ & $\begin{array}{l}\text { Rectangular } \\
\text { Duct } \\
200 \times 10 \times 2338\end{array}$ & $\begin{array}{l}\text { Cap } \\
\text { bubbly }\end{array}$ & $\begin{array}{l}J_{g}=0.095-0.59 \\
J_{l}=0.95-1.4\end{array}$ & $\begin{array}{l}\text { Ansys- } \\
\text { Fluent }\end{array}$ & & $\mathrm{k}-\varepsilon$ & & $\begin{array}{l}C_{D}-\text { Tomiyama, Sun } \\
C_{W L}-\text { Antal, } \\
C_{T D}-\text { Lahey, Lopez } \\
\quad \text { de Bertodano }\end{array}$ & & \\
\hline $\begin{array}{l}\text { Deju et al., } \\
(2013)^{8}\end{array}$ & $\begin{array}{l}\text { Pipe } \\
D=51.2 \\
L=3500 \\
D=195.3 \\
L=9000\end{array}$ & Bubbly & $\begin{array}{l}J_{g}=0.140-0.219 \\
J_{l}=1.017\end{array}$ & $\begin{array}{l}\text { Ansys CFX- } \\
11\end{array}$ & Euler & $\mathrm{k}-\omega \mathrm{SST}$ & $\begin{array}{l}\text { ABND, } \\
\text { MUSIG, } \\
\text { DQMOM, }\end{array}$ & $\begin{array}{l}C_{D} \text { - Ishii-Zuber, } \\
C_{L}-\text { Tomiyama, } \\
C_{W L}-\text { Antal } \\
C_{T D}-1\end{array}$ & $\begin{array}{l}\text { Yao and } \\
\text { Morel, } \\
\text { Prince and } \\
\text { Blanch }\end{array}$ & $\begin{array}{l}\text { Yao and Morel } \\
\text { Luo and } \\
\text { Svendson }\end{array}$ \\
\hline $\begin{array}{l}\text { Yeoh et al., } \\
(2012)^{9}\end{array}$ & $\begin{array}{l}\text { Pipe } \\
D=50.8 \\
L=3061\end{array}$ & Bubbly & $\begin{array}{l}J_{g}=0.0275-0.1130 \\
J_{l}=0.491-0.986\end{array}$ & $\begin{array}{l}\text { Ansys CFX- } \\
11\end{array}$ & Euler & $\mathrm{k}-\omega \mathrm{SST}$ & $\begin{array}{l}\text { DQMOM, } \\
\text { MUSIG }\end{array}$ & $\begin{array}{l}C_{D}-\text { Ishii-Zuber, } \\
C_{L}-\text { Tomiyama } \\
C_{W L}-\text { Antal, } \\
C_{T D}-\text { Burns }\end{array}$ & $\begin{array}{l}\text { Prince and } \\
\text { Blanch }\end{array}$ & $\begin{array}{l}\text { Luo and } \\
\text { Svendson }\end{array}$ \\
\hline $\begin{array}{l}\text { Cheung et } \\
\text { al., }(2012)^{10}\end{array}$ & $\begin{array}{l}\text { Pipe } \\
D=195.3 \\
L=9000\end{array}$ & $\begin{array}{l}\text { Bubbly, } \\
\text { cap } \\
\text { bubbly }\end{array}$ & $\begin{array}{l}J_{g}=0.0096-0.0898 \\
J_{l}=1.017\end{array}$ & $\begin{array}{l}\text { Ansys CFX- } \\
11\end{array}$ & Euler & $\mathrm{k}-\omega \mathrm{SST}$ & ABND & $\begin{array}{l}C_{D}-\text { Ishii-Zuber, } \\
C_{L}-\text { Tomiyama, } \\
C_{W L}-\text { Antal } \\
C_{T D}-\text { Burns }\end{array}$ & $\begin{array}{l}\text { Hibiki and } \\
\text { Ishii }\end{array}$ & Hibiki and Ishii \\
\hline $\begin{array}{l}\text { Deju et al., } \\
(2012)^{11}\end{array}$ & $\begin{array}{l}\text { Pipe } \\
D=51.2 \\
L=3500 \\
D=195.3 \\
L=9000\end{array}$ & Bubbly & $\begin{array}{l}J_{g}=0.140-0.219 \\
J_{l}=1.017\end{array}$ & $\begin{array}{l}\text { Ansys CFX- } \\
11\end{array}$ & Euler & $\mathrm{k}-\omega \mathrm{SST}$ & DQMOM & $\begin{array}{l}C_{D} \text { - Ishii-Zuber, } \\
C_{L}-\text { Tomiyama, } \\
C_{W L}-\text { Antal } \\
C_{T D}-\text { Burns }\end{array}$ & $\begin{array}{l}\text { Prince and } \\
\text { Blanch }\end{array}$ & $\begin{array}{l}\text { Luo and } \\
\text { Svendson }\end{array}$ \\
\hline $\begin{array}{l}\text { Liao et al., } \\
(2011)^{12}\end{array}$ & $\begin{array}{l}\text { Pipe } \\
D=195.3 \\
L=8000\end{array}$ & Bubbly & $\begin{array}{l}J_{g}=0.0368-0.0898 \\
J_{l}=0.405-1.017\end{array}$ & $\begin{array}{l}\text { Ansys CFX- } \\
12\end{array}$ & Eulerian & k- $\omega$ SST & MUSIG & $\begin{array}{l}C_{D} \text { - Grace } \\
C_{L}-\text { Tomiyama, } \\
C_{W L} \text { - Antal, } \\
C_{T D} \text { - Burns }\end{array}$ & $\begin{array}{l}\text { Prince and } \\
\text { Blanch }\end{array}$ & $\begin{array}{l}\text { Luo and } \\
\text { Svendson }\end{array}$ \\
\hline $\begin{array}{l}\text { Wang and } \\
\text { Sun } \\
(2011)^{13}\end{array}$ & $\begin{array}{l}\text { Pipe } \\
\mathrm{D}=48.3\end{array}$ & Bubbly & $\begin{array}{l}J_{g}=0.043-0.506 \\
J_{l}=0.064-2.34\end{array}$ & Fluent & Eulerian & & IATE & $\begin{array}{l}C_{D}-\text { Tomiyama } \\
C_{L}-\text { Tomiyama }\end{array}$ & $\begin{array}{l}\text { Kim, } \\
\text { Ishii }\end{array}$ & $\begin{array}{l}\text { Kim, } \\
\text { Ishii }\end{array}$ \\
\hline $\begin{array}{l}\text { Wang and } \\
\text { Sun } \\
(2011)^{14}\end{array}$ & $\begin{array}{l}\text { Rectangular } \\
\text { Duct } \\
200 \times 10 \\
\times 2950 \\
\end{array}$ & $\begin{array}{l}\text { Cap } \\
\text { bubbly }\end{array}$ & $\begin{array}{l}J_{g}=0.095-0.59 \\
J_{l}=0.95-1.4\end{array}$ & Fluent & & & IATE & & & \\
\hline $\begin{array}{l}\text { Pellacani et } \\
\text { al., }(2011)^{15}\end{array}$ & $\begin{array}{l}\text { Pipe } \\
D=52 \\
L=3340\end{array}$ & Bubbly & $\begin{array}{l}J_{g}=0.035-0.470 \\
J_{l}=0.51-2.036\end{array}$ & $\begin{array}{l}\text { Ansys CFX- } \\
12.1\end{array}$ & Eulerian & $\mathrm{k}-\omega \mathrm{SST}$ & IATE & $\begin{array}{l}C_{D}-\text { Grace } \\
C_{L}-\text { Tomiyama } \\
C_{W L}-\text { Antal } \\
C_{T D} \text { - Burns }\end{array}$ & Yao and Morel & Yao and Morel \\
\hline
\end{tabular}




\begin{tabular}{|c|c|c|c|c|c|c|c|c|c|c|}
\hline $\begin{array}{l}\text { Lo and } \\
\text { Zhang } \\
(2009)^{16}\end{array}$ & $\begin{array}{l}\text { Pipe } \\
\mathrm{D}=50.8 \\
\mathrm{~L}=3060\end{array}$ & Bubbly & $\begin{array}{l}J_{g}=0.321-0.624 \\
J_{l}=0.986-2.01\end{array}$ & $\begin{array}{l}\text { STAR-CD } \\
3.27\end{array}$ & Eulerian & k- $\varepsilon$ std. & & $\begin{array}{l}C_{D}-1.017 \\
C_{L}-(-0.2888) \\
C_{V M}-0.5\end{array}$ & $\begin{array}{l}\text { Newly } \\
\text { proposed } S_{\gamma} \\
\text { model }\end{array}$ & $\begin{array}{l}\text { Newly proposed } \\
S_{\gamma} \text { model }\end{array}$ \\
\hline $\begin{array}{l}\text { Sari et al., } \\
(2009)^{17}\end{array}$ & $\begin{array}{l}\text { Pipe } \\
D=38 \\
L=2800 \\
D=50.8 \\
L=3060 \\
D=57 \\
L=3060 \\
D=60 \\
L=2100\end{array}$ & Bubbly & $\begin{array}{l}J_{g}=0.027-0.275 \\
J_{l}=0.43-1.09\end{array}$ & $\begin{array}{l}\text { Fluent } \\
6.3 .26\end{array}$ & & k- $\varepsilon$ std. & $\begin{array}{l}\text { IATE, } \\
\text { BND }\end{array}$ & $\begin{array}{l}C_{D}-\text { Schiller-Naumann, } \\
\text { Ishii-Zuber, Tomiyama } \\
C_{L}-\text { Tomiyama, Ohunuki, } \\
\text { Frank, Moraga, Troshko, } \\
\text { Beyerlein, Behzadi, } \\
\text { Peterson } \\
C_{W L}-\text { Antal, Cheung } \\
C_{V M}-0.5\end{array}$ & $\begin{array}{l}\text { Hibiki-Ishii } \\
\text { Yao-Morel, } \\
\text { Wu }\end{array}$ & $\begin{array}{l}\text { Hibiki-Ishii, } \\
\text { Yao-Morel, } \\
\text { Wu }\end{array}$ \\
\hline $\begin{array}{l}\text { Wang and } \\
\text { Sun } \\
(2009)^{18}\end{array}$ & $\begin{array}{l}\text { Pipe } \\
D=50.8 \\
L=3060\end{array}$ & Bubbly & $\begin{array}{l}J_{g}=0.028-0.321 \\
J_{l}=0.491-0.986\end{array}$ & $\begin{array}{l}\text { Fluent } \\
6.2 .16\end{array}$ & Eulerian & $\mathrm{k}-\varepsilon$ std & IATE & $\begin{array}{l}C_{D}-\text { Tomiyama } \\
C_{L}=0 \text { and } 0.29\end{array}$ & Yao and Morel & Yao and Morel \\
\hline $\begin{array}{l}\text { Frank et al., } \\
(2008)^{19}\end{array}$ & $\begin{array}{l}\text { Pipe } \\
D=51.2 \\
L=4000 \\
D=194\end{array}$ & Bubbly & $\begin{array}{l}J_{g}=0.0040-0.0368 \\
J_{l}=0.225-1.611\end{array}$ & $\begin{array}{l}\text { Ansys CFX- } \\
10\end{array}$ & Eulerian & k- $\omega$ SST & MUSIG & $\begin{array}{l}C_{D}-\text { Grace and Tomiyama } \\
C_{L}-\text { Tomiyama } \\
C_{W L}-\text { Antal, Tomiyama, } \\
\quad \text { Frank } \\
C_{T D} \text { - Burns }\end{array}$ & $\begin{array}{l}\text { Prince and } \\
\text { Blanch }\end{array}$ & $\begin{array}{l}\text { Luo and } \\
\text { Svendson }\end{array}$ \\
\hline $\begin{array}{l}\text { Cheung et } \\
\text { al., }(2008)^{20}\end{array}$ & $\begin{array}{l}\text { Pipe } \\
\mathrm{D}=50.8 \\
\mathrm{~L}=3061\end{array}$ & $\begin{array}{l}\text { Bubbly } \\
\text { Bubbly to } \\
\text { slug }\end{array}$ & $\begin{array}{l}J_{g}=0.0473-0.242 \\
J_{l}=0.491-0.986\end{array}$ & $\begin{array}{l}\text { Ansys CFX- } \\
11\end{array}$ & & k- $\omega$ SST & MUSIG & & $\begin{array}{l}\text { Prince and } \\
\text { Blanch }\end{array}$ & $\begin{array}{l}\text { Luo and } \\
\text { Svendson }\end{array}$ \\
\hline $\begin{array}{l}\text { Krepper et } \\
\text { al., }(2008)^{21}\end{array}$ & $\begin{array}{l}\text { Pipe } \\
D=51.2 \\
L=3300\end{array}$ & Bubbly & $\begin{array}{l}J_{g}=0.14-0.2194 \\
J_{l}=1.017\end{array}$ & $\begin{array}{l}\text { Ansys CFX- } \\
4\end{array}$ & Euler & & MUSIG & $C_{L}$ - Tomiyama & $\begin{array}{l}\text { Prince and } \\
\text { Blanch }\end{array}$ & $\begin{array}{l}\text { Luo and } \\
\text { Svendson }\end{array}$ \\
\hline $\begin{array}{l}\text { Podila et } \\
\text { al., }(2007)^{22}\end{array}$ & pipe & Bubbly & $\begin{array}{l}J_{g}=0.113 \\
J_{l}=0.98\end{array}$ & $\begin{array}{l}\text { Fluent- } \\
6.3 .26\end{array}$ & Eulerian & $\mathrm{k}-\varepsilon$ & & $\begin{array}{l}C_{D}-\text { Ishii-Zuber, Rushe } \\
\quad \text { and Issa } \\
C_{L}-\text { Troshko } \\
C_{T D}-\text { Violet and Simonin } \\
C_{V M}-0.5 \\
\end{array}$ & $\begin{array}{l}\text { Prince and } \\
\text { Blanch, Luo, } \\
\text { Lehr }\end{array}$ & $\begin{array}{l}\text { Luo and } \\
\text { Svendson } \\
\text { Lehr }\end{array}$ \\
\hline $\begin{array}{l}\text { Cheung et } \\
\text { al., }(2007)^{23}\end{array}$ & $\begin{array}{l}\text { Pipe } \\
D=38 \\
L=2800 \\
D=50.8 \\
L=3061\end{array}$ & Bubbly & $\begin{array}{l}J_{g}=0.0270-0.1130 \\
J_{l}=0.491-1.087\end{array}$ & $\begin{array}{l}\text { Ansys CFX- } \\
10\end{array}$ & Eulerian & k- $\varepsilon$ std. & $\begin{array}{l}\text { ABND } \\
\text { MUSIG }\end{array}$ & $\begin{array}{l}C_{D}-\text { Ishii-Zuber } \\
C_{L}-\text { Tomiyama } \\
C_{W L}-\text { Antal } \\
C_{T D}-\text { Burns }\end{array}$ & $\begin{array}{l}\text { Hibiki-Ishii, } \\
\text { Yao-Morel, } \\
\text { Wu }\end{array}$ & $\begin{array}{l}\text { Hibiki-Ishii, } \\
\text { Yao-Morel, } \\
\text { Wu }\end{array}$ \\
\hline
\end{tabular}




\begin{tabular}{|c|c|c|c|c|c|c|c|c|c|c|}
\hline $\begin{array}{l}\text { Cheung et } \\
\text { al., }(2007)^{24}\end{array}$ & $\begin{array}{l}\text { Pipe } \\
D=38 \\
L=2800 \\
D=50.8 \\
L=3061\end{array}$ & Bubbly & $\begin{array}{l}J_{g}=0.0270-0.242 \\
J_{l}=0.491-1.087\end{array}$ & $\begin{array}{l}\text { Ansys CFX- } \\
10\end{array}$ & Eulerian & k- $\varepsilon$ std. & ABND & $\begin{array}{l}C_{D} \text { - Ishii-Zuber } \\
C_{L}-\text { Tomiyama } \\
C_{W L}-\text { Antal } \\
C_{T D}-\text { Burns }\end{array}$ & $\begin{array}{l}\text { Hibiki-Ishii, } \\
\text { Yao-Morel, } \\
\text { Wu, } \\
\text { Prince and } \\
\text { Blanch }\end{array}$ & $\begin{array}{l}\text { Hibiki-Ishii, } \\
\text { Yao-Morel, } \\
\text { Wu, } \\
\text { Luo and } \\
\text { Svendson }\end{array}$ \\
\hline $\begin{array}{l}\text { Huh et al., } \\
(2006)^{25}\end{array}$ & $\begin{array}{l}\text { Pipe } \\
D=80 \\
L=10000\end{array}$ & $\begin{array}{l}\text { Bubbly, } \\
\text { slug, } \\
\text { churn }\end{array}$ & $\begin{array}{l}J_{g}=0.1-0.698 \\
J_{l}=0.5-2\end{array}$ & & & & & & $\begin{array}{l}\text { Prince and } \\
\text { Blanch }\end{array}$ & $\begin{array}{l}\text { Prince and } \\
\text { Blanch }\end{array}$ \\
\hline $\begin{array}{l}\text { Frank } \\
(2005)^{26}\end{array}$ & $\begin{array}{l}\text { Pipe } \\
D=51.2 \\
L=4000\end{array}$ & Bubbly & $\begin{array}{l}J_{g}=0.004-0.0368 \\
J_{l}=0.225-1.611\end{array}$ & CFX-5.6 & Eulerian & k- $\omega$ SST & MUSIG & $\begin{array}{l}C_{D}-\text { Grace } \\
C_{L}-\text { Tomiyama } \\
C_{W L}-\text { Antal, Tomiyama, } \\
\text { Frank } \\
C_{T D}-\text { Burns, Lopez de } \\
\text { Bertodano }\end{array}$ & $\begin{array}{l}\text { Prince and } \\
\text { Blanch }\end{array}$ & $\begin{array}{l}\text { Luo and } \\
\text { Svendson }\end{array}$ \\
\hline $\begin{array}{l}\text { Yao and } \\
\text { Morel } \\
(2004)^{27}\end{array}$ & $\begin{array}{l}\text { Pipe } \\
D=38.1 \\
L=6000\end{array}$ & Bubbly & $\begin{array}{l}J_{g}=0.0588-0.1851 \\
J_{l}=0.877\end{array}$ & CATHRE & & $\mathrm{k}-\varepsilon$ & IATE & $\begin{array}{l}C_{D^{-}} \text {Ishii }(1990) \\
C_{L^{-}} 0.5 \\
C_{T D^{-}}\end{array}$ & $\begin{array}{l}\text { Wu, } \\
\text { Hibiki and } \\
\text { Ishii, } \\
\text { Hibiki and } \\
\text { Kim }\end{array}$ & $\begin{array}{l}\text { Wu, } \\
\text { Hibiki and Ishii, } \\
\text { Hibiki and Kim }\end{array}$ \\
\hline $\begin{array}{l}\text { Present } \\
\text { work }\end{array}$ & $\begin{array}{l}\text { Pipe } \\
\text { D = 25, 38, } \\
50.8,48.3 \\
51.2 \\
\text { L }=3000 \\
2922,3308\end{array}$ & Bubbly & $\begin{array}{l}J_{g}=0.0111-1.275 \\
J_{l}=0.4050-2.607\end{array}$ & Fluent & Eulerian & 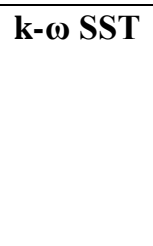 & Discrete & $\begin{array}{l}C_{D} \text { - Grace } \\
C_{L}-\text { Tomiyama } \\
C_{W L}-\text { Hosokawa } \\
C_{T D}-\text { Burns }\end{array}$ & $\begin{array}{l}\text { Brownian, } \\
\text { Abrahamson, } \\
\text { Luo }\end{array}$ & $\begin{array}{l}\text { Luo, Lehr. } \\
\text { Ghadiri, } \\
\text { Laakkonen }\end{array}$ \\
\hline
\end{tabular}

Table S3: Luo and Lehr breakage model parameters

\begin{tabular}{|l|l|l|l|l|}
\hline Models & $K\left(1 / \mathrm{m}^{3} \mathrm{sec}\right)$ & $n$ & $b$ & $m$ \\
\hline $\mathrm{Luo}^{28}$ & $0.9238 \varepsilon^{1 / 3} d^{-2 / 3} \alpha$ & $11 / 3$ & $12\left[f^{2 / 3}+(1-f)^{2 / 3}-1\right] \sigma \rho^{-1} \varepsilon^{-2 / 3} d^{-5 / 3} \beta^{-1}$ & $-11 / 3$ \\
\hline $\mathrm{Lehr}^{29}$ & $1.19 \varepsilon^{-1 / 3} d^{-7 / 3} \sigma \rho^{-1} f^{-1 / 3}$ & $13 / 3$ & $2 W e_{c r i t} \sigma \rho^{-1} \varepsilon^{-2 / 3} d^{-5 / 3} f^{-1 / 3}$ & $-2 / 3$ \\
\hline
\end{tabular}


Table S4: Model equations

\begin{tabular}{|c|c|c|}
\hline $\begin{array}{l}\text { Sr. } \\
\text { No. }\end{array}$ & Equation name & Equation expressions \\
\hline 1 & Continuity & $\frac{\partial\left(\alpha_{q} \rho_{q}\right)}{\partial t}+\nabla \cdot\left(\alpha_{q} \rho_{q} \overrightarrow{v_{q}}\right)=0$ \\
\hline 2 & Momentum & $\frac{\partial\left(\alpha_{q} \rho_{q} \overrightarrow{v_{q}}\right)}{\partial t}+\nabla \cdot\left(\alpha_{q} \rho_{q} \overrightarrow{v_{q}} \overrightarrow{v_{q}}\right)=-\alpha_{q} \nabla P+\nabla \cdot\left[\alpha_{G} \mu_{e, q}\left(\nabla \overrightarrow{v_{q}}+{\overrightarrow{v_{q}}}^{T}\right)\right]+F_{q}+\alpha_{q} \rho_{q} g$ \\
\hline 3 & Drag force & $\begin{array}{l}\overrightarrow{F_{D}}=C_{D}\left(\frac{\pi}{4} d_{b}^{2}\right) \frac{\rho_{l}}{2}\left(\overrightarrow{v_{g}}-\overrightarrow{v_{l}}\right)\left|\overrightarrow{v_{g}}-\overrightarrow{v_{l}}\right| \\
C_{D}=\max \left(\min \left(C_{D, \text { ellipse }}, C_{D, \text { cap }}\right), C_{D, \text { sphere }}\right) \\
C_{D, \text { sphere }}=\left\{\begin{array}{l}\frac{24}{\operatorname{Re}_{b}} \\
\frac{24\left(1+0.15 \mathrm{Re}_{b}^{0.687}\right)}{\operatorname{Re}_{b}}\end{array}\right. \\
C_{D, \text { ellipse }}=\frac{4 g d_{b}\left(\rho_{l}-\rho_{g}\right)}{3 U_{t}^{2} \rho_{l}} \\
C_{D, \text { cap }}=\frac{8}{3}\end{array}$ \\
\hline 4 & Lift force & $\overrightarrow{F_{L}}=-C_{L} \rho_{l} \alpha_{g}\left(\overrightarrow{v_{g}}-\overrightarrow{v_{l}}\right) \times\left(\nabla \times \overrightarrow{v_{l}}\right)$ \\
\hline
\end{tabular}




\begin{tabular}{|c|c|c|}
\hline & & $\begin{array}{l}C_{L}=\left\{\begin{array}{lc}\min \left[0.288 \tanh \left(0.121 \mathrm{Re}_{b}\right), f\left(E o^{\prime}\right)\right] & E o^{\prime} \leq 4 \\
f\left(E o^{\prime}\right) & 4<E o^{\prime} \leq 10 \\
-0.27 & 10<E o^{\prime}\end{array}\right. \\
f\left(E o^{\prime}\right)=0.00105 E o^{\prime 3}-0.0159 E o^{\prime 2}-0.0204 E o^{\prime}+0.474\end{array}$ \\
\hline 5 & $\begin{array}{l}\text { Wall lubrication } \\
\text { force }\end{array}$ & $\begin{array}{l}\overrightarrow{F_{W L}}=C_{W L} \rho_{l} \alpha_{g}\left|\left(v_{g}-v_{l}\right)_{\|}\right|^{2} \overrightarrow{n_{W}} \\
C_{W L}=C_{w} \max \left(0, \frac{1}{C_{w d}} \frac{1-\frac{y_{w}}{C_{w c} d_{b}}}{y_{w}\left(\frac{y_{w}}{C_{w c} d_{b}}\right)^{m-1}}\right)\end{array}$ \\
\hline 6 & $\begin{array}{l}\text { Turbulent } \\
\text { dispersion force }\end{array}$ & $\overrightarrow{F_{T D}}=-C_{T D} K_{g l} \frac{\mu_{l}^{t}}{\rho_{l} S c}\left(\frac{\nabla \alpha_{g}}{\alpha_{g}}-\frac{\nabla \alpha_{l}}{\alpha_{l}}\right)$ \\
\hline
\end{tabular}


Table S5: Experimental flow condition for wall and core peak case

\begin{tabular}{|c|c|c|c|c|c|c|}
\hline Case & Test cases & Pipe diameter (mm) & $J_{L}(\mathrm{~m} / \mathrm{s})$ & $J_{G}(\mathrm{~m} / \mathrm{s})$ & $\alpha_{G}(\%)$ & Measuring location (Z/D) \\
\hline \multirow[t]{2}{*}{ Wall peak } & $\begin{array}{c}\text { (Liu and Bankoff, } \\
1993)^{30}\end{array}$ & 38 & 1.087 & 0.027 & 2.42 & 36 \\
\hline & $\begin{array}{c}\text { (Hosokawa and } \\
\text { Tomiyama, 2009) }\end{array}$ & 25 & 0.5 & 0.018 & 2.31 & 68 \\
\hline Core peak & $\begin{array}{c}\text { (Liu and Bankoff, } \\
1993)^{30}\end{array}$ & 38 & 0.376 & 0.347 & 30.09 & 36 \\
\hline
\end{tabular}

References

(1) Dave, A. J.; Manera, A.; Beyer, M.; Lucas, D.; Bernard, M. Evaluation of Two-Group Interfacial Area Transport Equation Model for Vertical Small Diameter Pipes against High-Resolution Experimental Data. Chem. Eng. Sci. 2017, 162, $175-191$. https://doi.org/10.1016/j.ces.2017.01.001.

(2) Yuan, Y.; Li, X.; Tu, J. Numerical Modelling of Air--Nanofluid Bubbly Flows in a Vertical Tube Using the MUltiple-SIzeGroup (MUSIG) Model. Int. J. Heat Mass Transf. 2016, 102, 856-866.

(3) Peña-Monferrer, C.; Passalacqua, A.; Chiva, S.; Muñoz-Cobo, J. L. CFD Modelling and Validation of Upward Bubbly Flow in an Adiabatic Vertical Pipe Using the Quadrature Method of Moments. Nucl. Eng. Des. 2016, 301, 320-332. https://doi.org/10.1016/j.nucengdes.2016.03.006.

(4) Swiderski, K.; Narayanan, C.; Lakehal, D. Application of N-Phase Algebraic Slip Model and Direct Quadrature Method of Moments to the Simulation of Air-Water Flow in Vertical Risers and Bubble Column Reactor. Comput. Chem. Eng. 2016, 90, 151-160. https://doi.org/10.1016/j.compchemeng.2016.04.023.

(5) Deju, L.; Cheung, S. C. P.; Yeoh, G. H.; Qi, F.; Tu, J. Comparative Analysis of Coalescence and Breakage Kernels in Vertical Gas-Liquid Flow. Can. J. Chem. Eng. 2015, 93 (7), 1295-1310. https://doi.org/10.1002/cjce.22196. 
(6) Schlegel, J. P.; Hibiki, T.; Ishii, M. Two-Group Modeling of Interfacial Area Transport in Large Diameter Channels. Nucl. Eng. Des. 2015, 293, 75-86.

(7) Wang, X.; Sun, X. Numerical Simulations of Air--Water Cap-Bubbly Flows Using Two-Group Interfacial Area Transport Equation. Ann. Nucl. Energy 2014, 71, 399-410.

(8) Deju, L.; Cheung, S. C. P.; Yeoh, G. H.; Tu, J. Y. Capturing Coalescence and Break-up Processes in Vertical Gas-Liquid Flows: Assessment of Population Balance Methods. Appl. Math. Model. 2013, 37 (18-19), 8557-8577. https://doi.org/10.1016/j.apm.2013.03.063.

(9) Yeoh, G. H.; Cheung, S. C. P.; Tu, J. Y. On the Prediction of Bubble Size Distribution and Void Fraction in Vertical GasLiquid Flows. J. Comput. Multiph. Flows 2012, 4 (1), 1-22.

(10) Cheung, S. C. P.; Yeoh, G. H.; Qi, F. S.; Tu, J. Y. Classification of Bubbles in Vertical Gas--Liquid Flow: Part 2--A Model Evaluation. Int. J. Multiph. Flow 2012, 39, 135-147.

(11) Deju, L.; Cheung, S. C. P.; Yeoh, G. H.; Tu, J. Study of Isothermal Vertical Bubbly Flow Using Direct Quadrature Method of Moments. J. Comput. Multiph. Flows 2012, 4 (1). https://doi.org/10.1260/1757-482X.4.1.23.

(12) Liao, Y.; Lucas, D.; Krepper, E.; Schmidtke, M. Development of a Generalized Coalescence and Breakup Closure for the Inhomogeneous MUSIG Model. Nucl. Eng. Des. 2011, 241 (4), 1024-1033.

(13) Wang, X.; Sun, X. Effects of Non-Uniform Inlet Boundary Conditions and Lift Force on Prediction of Phase Distribution in Upward Bubbly Flows with Fluent-IATE. Nucl. Eng. Des. 2011, 241 (7), 2500-2507.

(14) Wang, X.; Sun, X. CFD Simulations of Cap-Bubbly Two-Phase Flows Using Two-Group Interfacial Area Transport Equation. In ASME-JSME-KSME 2011 Joint Fluids Engineering Conference; 2011; pp 1315-1330.

(15) Pellacani, F.; Chiva, S.; Peña, C.; Macián, R. Implementation of a One-Group Interfacial Area Transport Equation in a CFD Code for the Simulation of Upward Adiabatic Bubbly Flow. 2011.

(16) Lo, S.; Zhang, D. Modelling of Break-up and Coalescence in Bubbly Two-Phase Flows. J. Comput. Multiph. Flows 2009, 1 (1), 23-38.

(17) Sari, S.; Ergün, Ş.; Barik, M.; Kocar, C.; Sökmen, C. N. Modeling of Isothermal Bubbly Flow with I $\{$ dotless $\}$ nterfacial Area Transport Equation and Bubble Number Density Approach. Ann. Nucl. Energy 2009, 36 (2), 222-232. 
https://doi.org/10.1016/j.anucene.2008.11.016.

(18) Wang, X.; Sun, X. CFD Simulation of Phase Distribution in Adiabatic Upward Bubbly Flows Using Interfacial Area Transport Equation. Nucl. Technol. 2009, 167 (1), 71-82.

(19) Frank, T.; Zwart, P.; Krepper, E.; Prasser, H. M.; Lucas, D. Validation of CFD Models for Mono-and Polydisperse Air-water Two-Phase Flows in Pipes. Nucl. Eng. Des. 2008, 238 (3), 647-659. https://doi.org/10.1016/j.nucengdes.2007.02.056.

(20) Cheung, S. C. P.; Yeoh, G. H.; Tu, J. Y. On the Modelling of Population Balance in Isothermal Vertical Bubbly Flows-average Bubble Number Density Approach. Chem. Eng. Process. Process Intensif. 2007, 46 (8), 742-756.

(21) Krepper, E.; Lucas, D.; Frank, T.; Prasser, H. M.; Zwart, P. J. The Inhomogeneous MUSIG Model for the Simulation of Polydispersed Flows. Nucl. Eng. Des. 2008, 238 (7), 1690-1702. https://doi.org/10.1016/j.nucengdes.2008.01.004.

(22) Podila, K.; Al Taweel, A. M.; Koksal, M.; Troshko, A.; Gupta, Y. P. CFD Simulation of Gas--Liquid Contacting in Tubular Reactors. Chem. Eng. Sci. 2007, 62 (24), 7151-7162.

(23) Cheung, S. C. P.; Deju, L.; Yeoh, G. H.; Tu, J. Y. Modeling of Bubble Size Distribution in Isothermal Gas--Liquid Flows: Numerical Assessment of Population Balance Approaches. Nucl. Eng. Des. 2013, 265, 120-136.

(24) Cheung, S. C. P.; Yeoh, G. H.; Tu, J. Y. On the Numerical Study of Isothermal Vertical Bubbly Flow Using Two Population Balance Approaches. Chem. Eng. Sci. 2007, 62 (17), 4659-4674.

(25) Huh, B. G.; Euh, D. J.; Yoon, H. Y.; Yun, B. J.; Song, C. H.; Chung, C. H. Mechanistic Study for the Interfacial Area Transport Phenomena in an Air/Water Flow Condition by Using Fine-Size Bubble Group Model. Int. J. Heat Mass Transf. 2006, 49 (2122), 4033-4042. https://doi.org/10.1016/j.ijheatmasstransfer.2005.11.037.

(26) Frank, T. Advances in Computational Fluid Dynamics (CFD) of 3-Dimensional Gas-Liquid Multiphase Flows. In NAFEMS Seminar: Simulation of Complex Flows (CFD)--Applications and Trends, Wiesbaden, Germany; 2005; pp 1-18.

(27) Yao, W.; Morel, C. Volumetric Interfacial Area Prediction in Upward Bubbly Two-Phase Flow. Int. J. Heat Mass Transf. 2004, $47(2), 307-328$.

(28) Luo, H. Coalescence, Breakup and Liquid Circulation in Bubble Column Reactors. Dr. Ing, Thesis, Department of Chemical Engineering, The Norwegian Institute of technology, 1993.

(29) Lehr, F.; Millies, M.; Mewes, D. Bubble-Size Distributions and Flow Fields in Bubble Columns. AIChE J. 2002, 48 (11), 2426- 
2443.

(30) Liu, T. J.; Bankoff, S. G. Structure of Air-Water Bubbly Flow in a Vertical Pipe-II. Void Fraction, Bubble Velocity and Bubble Size Distribution. Int. J. Heat Mass Transf. 1993, 36 (4), 1061-1072. https://doi.org/10.1016/S0017-9310(05)80290-X.

(31) Hosokawa, S.; Tomiyama, A. Multi-Fluid Simulation of Turbulent Bubbly Pipe Flows. Chem. Eng. Sci. 2009, 64 (24), 53085318 . 